\title{
Association between chronic cerebrospinal venous insufficiency and multiple sclerosis: a meta-analysis
}

\author{
Andreas Laupacis MD MSc, Erin Lillie MSc, Andrew Dueck MD MSc, Sharon Straus MD MSc, \\ Laure Perrier MEd MLIS, Jodie M. Burton MD MSc, Richard Aviv MBChB, Kevin Thorpe MMath, \\ Thomas Feasby MD, Julian Spears MD SM
}

See related commentary by Fox at www.cmaj.ca/lookup/doi/10.1503/cmaj.111506

\begin{abstract}
Background: It has been proposed by Zamboni and colleagues that multiple sclerosis is caused by chronic cerebrospinal venous insufficiency, a term used to describe ultrasound-detectable abnormalities in the anatomy and flow of intra- and extracerebral veins. We conducted a meta-analysis of studies that reported the frequency of chronic cerebrospinal venous insufficiency among patients with and those without multiple sclerosis.
\end{abstract}

\section{Methods: We searched MEDLINE and EMBASE as well as bibliographies of relevant articles for eligible studies. We included studies if they used ultrasound to diagnose chronic cerebrospinal venous insufficiency and com- pared the frequency of the venous abnormali- ties among patients with and those without multiple sclerosis.}

Results: We identified eight eligible studies: all included healthy controls, and four of them also included a control group of patients with neurologic diseases other than multiple sclerosis. Chronic cerebrospinal venous insufficiency was more frequent among patients with multi-
$\mathrm{M}$ ultiple sclerosis is a chronic demyelinating and degenerative disease of the central nervous system. The exact cause remains unknown, but most evidence favours an autoimmune mechanism. ${ }^{1}$ In 2006, Zamboni and colleagues proposed that multiple sclerosis is caused by abnormalities in the direction and pathway of cerebral venous flow, leading to deposition of iron in the brain, which triggers an autoimmune reaction. ${ }^{2}$ They reported that patients with multiple sclerosis had a higher frequency of abnormalities of anatomy and flow in the internal jugular, deep cerebral, vertebral and azygous veins than individuals without multiple sclerosis had., ${ }^{3,4}$ They called this condition chronic cerebrospinal venous insufficiency. They further described detection of this ple sclerosis than among the healthy controls (odds ratio [OR] 13.5, 95\% confidence interval [CI] 2.6-71.4), but there was extensive unexplained heterogeneity among the studies. The association remained significant in the most conservative sensitivity analysis (OR 3.7, 95\% Cl 1.2-11.0), in which we removed the initial study by Zamboni and colleagues and added a study that did not find chronic cerebrospinal venous insufficiency in any patient. Although chronic cerebrospinal venous insufficiency was also more frequent among patients with multiple sclerosis than among controls with other neurologic diseases (OR 32.5, 95\% Cl 0.6-1775.7), the association was not statistically significant, the 95\% Cl was wide, and the OR was less extreme after removal of the study by Zamboni and colleagues (OR 3.5, 95\% 0.8-15.8).

Interpretation: Our findings showed a positive association between chronic cerebrospinal venous insufficiency and multiple sclerosis. However, poor reporting of the success of blinding and marked heterogeneity among the studies included in our review precluded definitive conclusions.
Competing interests:

Andreas Laupacis receives honoraria as a member of a data safety monitoring board for studies of two drugs for multiple sclerosis funded by Novartis

Pharmaceuticals. Jodie Burton has received unrestricted educational support and honoraria for speaking and educational engagements from Teva

Neuroscience Canada, EMD Serono and Biogen Idec Canada. No competing interests declared by the other authors.

Disclaimer: Andreas Laupacis is a member of CMAJ's Editorial Board, and Sharon Straus is an associate editor for $C M A J$. Neither was involved in the editorial decision-making process for this article.

This article has been peer reviewed.

Correspondence to: Dr. Andreas Laupacis, laupacisa@smh.ca

condition by means of transcranial and extracranial Doppler ultrasonography. This method of detection requires the evaluation of five ultrasound parameters that assess both venous blood flow and anatomy. ${ }^{3,5}$ Chronic cerebrospinal venous insufficiency is diagnosed if a patient has an abnormality in two or more of the five parameters.

There is controversy about the frequency and role of chronic cerebrospinal venous insufficiency in patients with multiple sclerosis ${ }^{6,7}$ and whether the frequency differs between patients with and those

A summary for patients that explains the purpose, design and main findings of this study is available in Appendix 1, at www.cmaj.ca/lookup/suppl /doi:10.1503/cmaj.111074/-/DC1. 
without multiple sclerosis. We performed a systematic review and meta-analysis of all peer-reviewed reports of studies that compared the frequency of chronic cerebrospinal venous insufficiency among patients with and those without multiple sclerosis.

\section{Methods}

\section{Literature search}

We searched the MEDLINE and EMBASE electronic databases for articles reporting the frequency of chronic cerebrospinal venous insufficiency among patients with and those without multiple sclerosis published from 2005 to June 28,2011 . No language restrictions were imposed. The search was done as part of a larger

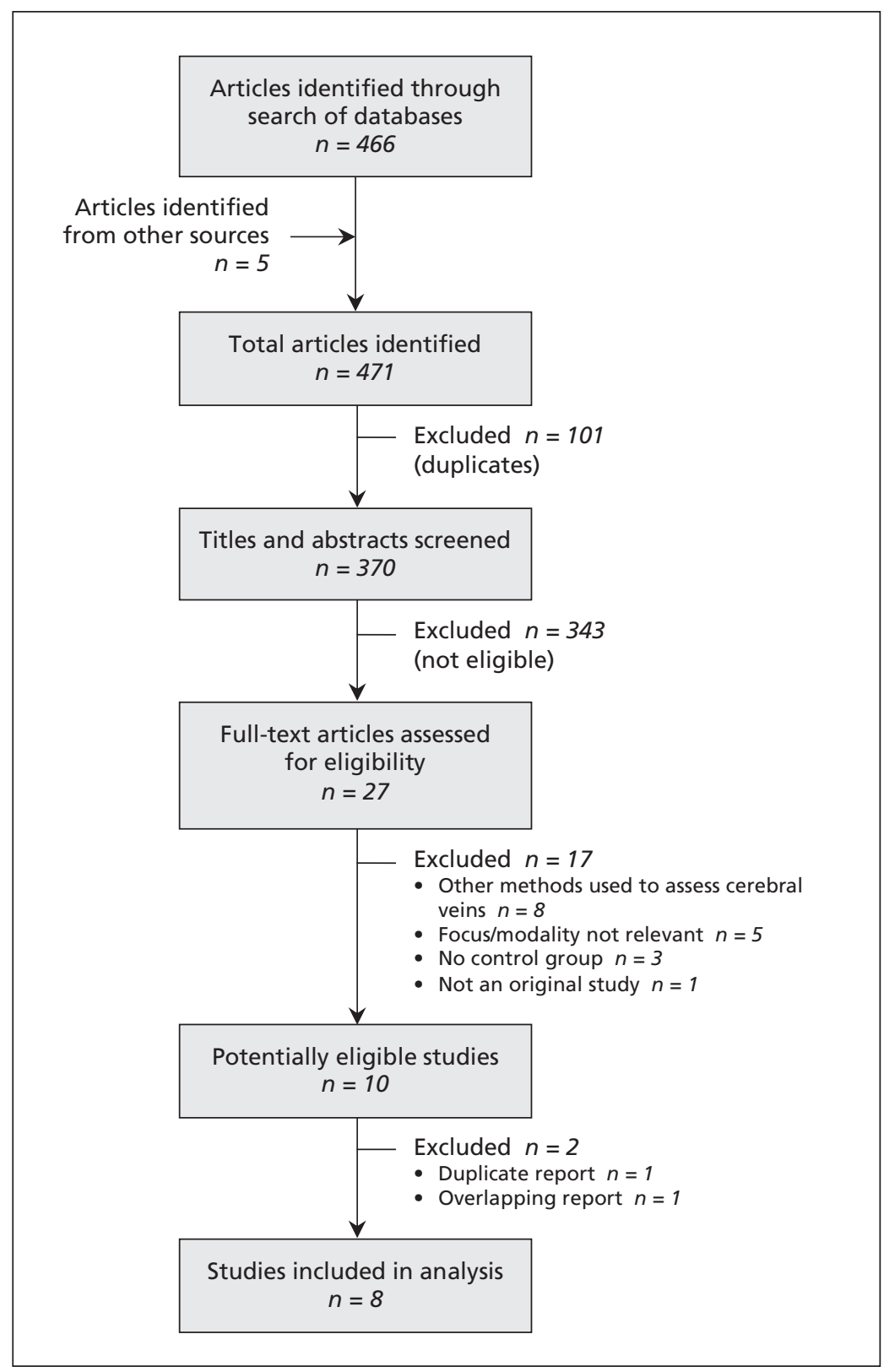

Figure 1: Results of literature search and identification of eligible studies. systematic review of all methods of evaluating the cerebral and azygos veins in patients with multiple sclerosis. The detailed search strategy, carried out by an information scientist (L.P.), is shown in Appendix 1 (available at www.cmaj.ca /lookup/suppl/doi:10.1503/cmaj.111074/-/DC1). We examined reference lists of all articles that met the eligibility criteria and of review articles to identify studies that may have been missed by the database searches. We also contacted experts to verify that no relevant studies were omitted.

\section{Study selection}

Studies were eligible if they met all of the following criteria: they reported original data in a peerreviewed publication; they used Doppler ultrasonography to detect chronic cerebrospinal venous insufficiency; and they assessed patients who had multiple sclerosis and had at least one control group (healthy controls or patients with neurologic diseases other than multiple sclerosis).

The titles and abstracts of all studies identified were screened independently by two of us (A.L. and S.S.). Studies that appeared to be relevant were selected and the full-text versions assessed independently by the same two reviewers. Disagreements were resolved by consensus or involvement of a third reviewer (A.D.).

\section{Data extraction and analysis}

Two reviewers independently extracted data about the study characteristics, the methodologic quality of the studies (assessed using items derived from the Newcastle-Ottawa Quality Assessment Scale tool for observational studies ${ }^{8}$ ), patient characteristics and ultrasound results. Disagreements were resolved by consensus.

We used the Cochrane Review Manager 5.1, version 5.1.2, to generate odds ratios and forest plots, to determine whether there was a statistical association between chronic cerebrospinal venous insufficiency and multiple sclerosis (using a random-effects model) and to assess heterogeneity of the studies. ${ }^{9}$ Heterogeneity was quantified using the $I^{2}$ statistic.

To test the robustness of the main results, we conducted sensitivity analyses, which included the removal of the initial study by Zamboni and colleagues $^{4}$ and the addition of a study by Doepp and colleagues ${ }^{10}$ that did not find chronic cerebrospinal venous insufficiency in any patient. ${ }^{10}$

\section{Results}

\section{Identification of eligible studies}

Of the 471 records found initially, we identified 10 studies that used Doppler ultrasonography and met our inclusion criteria (Figure 1). Of 
three studies from Zamboni's group, ${ }^{3-5}$ two described the same patients and reported the same results, ${ }^{3,5}$ and two appeared to describe overlapping patients ${ }^{3,4}$ but did not report dates of enrolment. We included what appeared to be the most recent study by Zamboni and colleagues with the largest number of patients ${ }^{4}$ and excluded the other two studies. Thus, eight studies were included in the meta-analysis., ${ }^{410-16}$

\section{Study characteristics}

The studies were conducted in Italy $(n=3)$, Germany $(n=3)$, Jordan $(n=1)$ and the United States $(n=1)$. Most enrolled a small number of patients with multiple sclerosis (range 10-310) and controls (range 7-210) (Tables 1, 2 and 3). All eight studies included healthy controls, and four also included controls with neurologic diseases other than multiple sclerosis. All studies except that by Centonze and coworkers ${ }^{13}$ described their assessment of the five ultrasound parameters for chronic cerebrospinal venous insufficiency as well as the ultimate diagnosis of the condition. Both Zamboni and colleagues ${ }^{4}$ and Krogias and coworkers ${ }^{14}$ included healthy individuals and patients with neurologic diseases other than multiple sclerosis as controls, but they did not report the results for these two groups separately. No controls in either of these studies had chronic cerebrospinal venous insufficiency. However, for the analysis of the individual ultrasound parameters, if one or more controls in the study by Zamboni and colleagues fulfilled the criteria for a parameter, we considered all of the controls to be healthy controls in the analysis of that parameter because $75 \%$ of their controls were healthy controls. Similarly, if one or more of the controls in the study by Krogias and coworkers fulfilled the criteria for a parameter, we considered all of the controls to be patients with other neurologic diseases in the analysis of that parameter because $71 \%$ of their controls had such diseases.

Most of the studies did not describe clearly how patients were identified for enrolment and

Table 1: Characteristics of studies included in meta-analysis that compared the frequency of chronic cerebrospinal venous insufficiency among patients with and without multiple sclerosis (MS)

\begin{tabular}{|c|c|c|c|c|c|}
\hline \multirow[b]{2}{*}{ Study } & \multirow{2}{*}{$\begin{array}{l}\text { No. of } \\
\text { patients } \\
\text { with MS }\end{array}$} & \multicolumn{2}{|r|}{ No. of controls } & \multirow[b]{2}{*}{ Ultrasound equipment used } & \multirow[b]{2}{*}{ Blinding } \\
\hline & & $\begin{array}{l}\text { Healthy } \\
\text { controls }\end{array}$ & $\begin{array}{l}\text { Patients with neurologic } \\
\text { diseases other than MS }\end{array}$ & & \\
\hline Al-Omari et al. ${ }^{11}$ & 25 & 25 & NA & $\begin{array}{l}\text { Philips ATL HDI } 5000 \text { with } \\
\text { 4-7-MHz linear probe and/or } \\
\text { 5-8-Mhz curved probe; } \\
\text { transcranial exam not performed } \\
\text { (probe not available) }\end{array}$ & Not blinded \\
\hline Baracchini et al. ${ }^{12}$ & 50 & 110 & 60 & $\begin{array}{l}\text { Philips iU22 xMatrix; } 5-10-\mathrm{MHz} \\
\text { linear array probe for } \\
\text { extracranial exam, } 1-3-\mathrm{MHz} \\
\text { phased array probe for } \\
\text { transcranial exam }\end{array}$ & $\begin{array}{l}\text { Described as blinded but did } \\
\text { not describe how blinding } \\
\text { was ensured or whether } \\
\text { blinding was tested }\end{array}$ \\
\hline Centonze et al. ${ }^{13}$ & 84 & 56 & NA & $\begin{array}{l}\text { Esaote Biomedica MyLab-Vinco } \\
25 \text { with 11-3-MHz LA322 linear } \\
\text { probe }\end{array}$ & $\begin{array}{l}\text { Described blinding process but } \\
\text { did not describe whether } \\
\text { blinding was achieved }\end{array}$ \\
\hline Doepp et al. ${ }^{10}$ & 56 & 20 & NA & $\begin{array}{l}\text { Toshiba PowerVision 6000; } \\
\text { 7.5-MHz linear transducer for } \\
\text { extracranial exam, 2.5-MHz probe } \\
\text { for transcranial exam }\end{array}$ & Not blinded \\
\hline Krogias et al. ${ }^{14}$ & 10 & 2 & 5 & Not described & Not blinded \\
\hline Mayer et al. ${ }^{15}$ & 20 & 20 & NA & $\begin{array}{l}\text { Phillips iU22 with L9-3 probe to } \\
\text { assess internal jugular veins and } \\
\text { vertebral veins; S5-1 probe used } \\
\text { to assess intracranial veins }\end{array}$ & $\begin{array}{l}\text { Described blinding process } \\
\text { but did not describe whether } \\
\text { blinding was achieved }\end{array}$ \\
\hline Zamboni et al. ${ }^{4}$ & 109 & 132 & 45 & $\begin{array}{l}\text { Esoate Biosound MyLab 25; } \\
\text { 7.5-10 MHz for extracranial } \\
\text { exam, } 2.5 \mathrm{MHz} \text { for intracranial } \\
\text { exam }\end{array}$ & $\begin{array}{l}\text { Described as blinded but did } \\
\text { not describe how blinding } \\
\text { was undertaken or whether } \\
\text { blinding was achieved }\end{array}$ \\
\hline Zivadinov et al. ${ }^{16}$ & 310 & 163 & 26 & $\begin{array}{l}\text { Esaote-Biosound MyLab } \\
\text { Gold } 25 \text { equipped with } 2.5- \\
\text { and } 7.5-10-\mathrm{MHz} \text { transducers }\end{array}$ & $\begin{array}{l}\text { Described blinding process but } \\
\text { did not describe whether } \\
\text { blinding was achieved }\end{array}$ \\
\hline
\end{tabular}


appeared to enrol a convenience sample (Table 4). Three studies were not blinded (Table 1). The method of blinding of the ultrasound technician and the interpreter of the images was poorly described in two studies, ${ }^{4,12}$ reasonably welldescribed in two others ${ }^{13,16}$ and well-described in one study. ${ }^{15}$ However, the success of blinding was not reported by any study.

Although one study described the ultrasound technique used in great detail, ${ }^{16}$ the training of ultrasonographers and radiologists in assessing this relatively novel entity, and in ensuring quality control, was generally poorly described (Table 4). Two of the studies indicated that their ultrasonographers were trained by Zamboni's group. ${ }^{13,16}$ No study described a run-in period during which intra- and interobserver reliability was determined to be good, although one study described intraobserver variability. ${ }^{16}$

Most of the patients with multiple sclerosis had relapsing-remitting disease; those in the study by Baracchini and colleagues ${ }^{12}$ had clinically isolated syndrome (Table 2). Most patients were taking disease-modifying agents. The mean age, proportion of women and mean Expanded Disability Status Scale scores (range 1.5-5.8) were typical of patients with clinically isolated syndrome and multiple sclerosis (Table 2).

\section{Frequency of chronic cerebrospinal venous insufficiency}

The diagnosis of chronic cerebrospinal venous insufficiency requires that a patient have an abnormality in at least two of the following five ultrasound parameters: ${ }^{3,4}$ (1) reflux in the internal jugular veins or vertebral veins for more than 0.88 seconds, assessed in the supine and sitting position without use of the Valsalva manoeuvre;
(2) reversal of flow in at least one of the deep cerebral veins; (3) stenoses of the internal jugular vein identified on high-resolution B-mode imaging; (4) flow in the internal jugular veins or vertebral veins not detectable by Doppler ultrasonography; and (5) reverted postural control of the main cerebral venous outflow pathway (internal jugular veins). [A diagram of the five vascular abnormalities is available online at www .cmaj.ca/lookup/suppl/doi:10.1503/cmaj.111506 /-/DC1. - Ed.]

Figure 2 displays the results of the metaanalyses of data on the frequency of chronic cerebrospinal venous insufficiency among patients with multiple sclerosis compared with healthy controls and with controls who had a neurologic disease other than multiple sclerosis.

In the comparisons with healthy controls, the frequency of chronic cerebrospinal venous insufficiency among patients with multiple sclerosis varied markedly, from none ${ }^{10,15}$ to $100 \% 4$ (Figure 2). The association between chronic cerebrospinal venous insufficiency and multiple sclerosis also varied markedly, from an odds ratio (OR) of 0.32 (95\% CI 0.01-8.3) to an OR of 58035.0 (95\% CI 1142.22948 755.8). The pooled analysis revealed a statistically significant association between chronic cerebrospinal venous insufficiency and multiple sclerosis, as compared with healthy controls (OR 13.5, 95\% CI 2.6-71.4); however, there was extensive heterogeneity among study results $\left(I^{2}=89 \%\right)$.

In terms of the five individual parameters, patients with multiple sclerosis had significantly higher odds than healthy controls of having reflux in the internal jugular or vertebral veins (OR 13.7, 95\% CI 2.0-93.8), although there was

Table 2: Characteristics of patients with multiple sclerosis (MS) included in studies

\begin{tabular}{|c|c|c|c|c|c|c|c|c|}
\hline \multirow[b]{2}{*}{ Study } & \multicolumn{3}{|c|}{ Type of MS; no. of MS patients } & \multirow[b]{2}{*}{ Age, yr } & \multirow[b]{2}{*}{$\begin{array}{c}\text { Female, } \\
\%\end{array}$} & \multirow[b]{2}{*}{$\begin{array}{l}\text { EDSS } \\
\text { score }\end{array}$} & \multirow{2}{*}{$\begin{array}{l}\text { Use of disease- } \\
\text { modifying } \\
\text { medications, \% }\end{array}$} & \multirow[b]{2}{*}{$\begin{array}{l}\text { Duration } \\
\text { of MS, } \mathrm{yr}\end{array}$} \\
\hline & $\begin{array}{l}\text { Clinically isolated } \\
\text { syndrome }\end{array}$ & $\begin{array}{l}\text { Relapsing- } \\
\text { remitting }\end{array}$ & Other & & & & & \\
\hline Al-Omari et al. ${ }^{11}$ & 0 & 21 & 4 & $35^{*}$ & 52 & NA & NA & NA \\
\hline Baracchini et al. ${ }^{12}$ & 50 & 0 & 0 & $33^{*}$ & 70 & $1.5 t$ & 28 & NA \\
\hline Centonze et al..$^{13}$ & 0 & 69 & 15 & $39 *$ & 62 & NA & 82 & NA \\
\hline Krogias et al. ${ }^{14}$ & 0 & 2 & 8 & $42 \dagger$ & 30 & $5.8+$ & NA & NA \\
\hline Mayer et al. ${ }^{15}$ & 0 & 17 & 3 & $42^{*}$ & 65 & $3 \dagger$ & 90 & $13^{*}$ \\
\hline 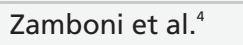 & 0 & 69 & 40 & $40+$ & 59 & $2+$ & NA & $6+$ \\
\hline Zivadinov et al. ${ }^{16}$ & 21 & 191 & 98 & $48 \dagger$ & 76 & $3 \dagger$ & $89 \ddagger$ & $12 \dagger$ \\
\hline
\end{tabular}


extensive heterogeneity among study results $\left(I^{2}=\right.$ $85 \%$ ). The other parameters were also more frequent among patients with multiple sclerosis than among healthy controls, but these trends did not reach statistical significance (Figure 3).

In the comparisons with controls who had other neurologic diseases, chronic cerebrospinal venous insufficiency was more frequent among patients with multiple sclerosis (Figure 2), but the association was not statistically significant (OR 32.5, 95\% CI 0.59-1775.7) and heterogeneity among the study results was large $\left(I^{2}=90 \%\right)$. Again, the only individual ultrasound parameter that was significantly more frequent among patients with multiple sclerosis than among controls with other neurologic diseases was reflux in the internal jugular or vertebral veins (OR 6.7, 95\% CI $1.3-34.8 ; I^{2}=73 \%$ ) (Figure 4$)$.

\section{Sensitivity analyses}

Because Zamboni and colleagues were the first to describe chronic cerebrospinal venous insufficiency (and therefore their study could be considered hypothesis-generating) and because the OR found in their study was extremely high, ${ }^{4}$ we repeated the meta-analysis with their study removed. The odds of chronic cerebrospinal venous insufficiency being more frequent among patients with multiple sclerosis than among healthy controls decreased from an OR of 13.5 to 4.7 (95\% 1.5-14.6), but the association remained statistically significant with considerable heterogeneity $\left(I^{2}=73 \%\right)$ (Figure A in Appendix 2, available at www.cmaj.ca/lookup/suppl/doi: 10.1503/cmaj.111074/-/DC1).

When we repeated the meta-analysis after changing zero to one in each cell for the study by Doepp and colleagues, in which none of the patients with multiple sclerosis or healthy controls had chronic cerebrospinal venous insufficiency, the results were similar to those of the primary analysis (OR 8.8, 95\% CI 1.9-42.0; $I^{2}=$ $88 \%$ ) (Figure B in Appendix 2).

Table 3: Characteristics of participants included in control groups

\begin{tabular}{|c|c|c|c|}
\hline Study & $\begin{array}{c}\text { No. of } \\
\text { participants }\end{array}$ & Age, yr & Female, $\%$ \\
\hline \multicolumn{4}{|l|}{ Healthy controls } \\
\hline Al-Omari et al. ${ }^{11}$ & 25 & $34 \ddagger$ & 52 \\
\hline \multicolumn{4}{|l|}{ Baracchini et al. $^{12}$} \\
\hline Group 1* & 50 & $33 \ddagger$ & 70 \\
\hline Group 2† & 60 & $63 \ddagger$ & 53 \\
\hline Centonze et al. ${ }^{13}$ & 56 & $42 \ddagger$ & 64 \\
\hline Doepp et al. ${ }^{10}$ & 20 & $41 \ddagger$ & 60 \\
\hline Mayer et al. ${ }^{15}$ & 20 & $34 \ddagger$ & 50 \\
\hline \multicolumn{4}{|l|}{ Zamboni et al. ${ }^{4}$} \\
\hline Group 1* & 60 & $37 \S$ & 53 \\
\hline Group $2 \dagger$ & 72 & $58 \S$ & 60 \\
\hline Zivadinov et al. $^{16}$ & 163 & $47 \S$ & 54 \\
\hline \multicolumn{4}{|c|}{$\begin{array}{l}\text { Controls with neurologic } \\
\text { diseases other than MS }\end{array}$} \\
\hline Baracchini et al. ${ }^{12}$ & 60 & $64 \ddagger$ & 53 \\
\hline Krogias et al. ${ }^{14}$ & 7 & $40 \S$ & 29 \\
\hline Zamboni et al. ${ }^{4}$ & 45 & $60 \S$ & 44 \\
\hline Zivadinov et al. $^{16}$ & 26 & $50 \S$ & 73 \\
\hline \multicolumn{4}{|c|}{$\begin{array}{l}\text { Note: MS = multiple sclerosis, NA = not applicable. } \\
\text { *Healthy controls in group } 1 \text { were matched with MS patients. } \\
\text { tIn the study by Baracchini et al., healthy controls in group } 2 \text { were matched with controls } \\
\text { who had neurologic diseases other than MS; in the study by Zamboni et al., healthy controls } \\
\text { in group } 2 \text { were older than the median age of the European MS population. } \\
\text { fMean. } \\
\text { SMedian. }\end{array}$} \\
\hline
\end{tabular}

Table 4: Methodologic quality of studies included in meta-analysis*

\begin{tabular}{|c|c|c|c|c|c|c|}
\hline Study & \multicolumn{2}{|c|}{ Experience level } & \multicolumn{2}{|c|}{ Case definition } & \multicolumn{2}{|c|}{ Representativeness of patients } \\
\hline Al-Omari et al. ${ }^{11}$ & No & No & Not sure & No & Convenience & Yes \\
\hline Centonze et al. ${ }^{13}$ & Yes & Yes & Yes & No & Convenience & Yes \\
\hline Doepp et al. ${ }^{10}$ & No & No & Yes & No & Convenience & Yes \\
\hline Krogias et al. $^{14}$ & No & No & Not sure & No & Convenience & Not sure \\
\hline Mayer et al. ${ }^{15}$ & No & No & Not sure & No & Convenience & No \\
\hline Zamboni et al. ${ }^{4}$ & Yes & Yes & Not sure & No & Convenience & Yes \\
\hline
\end{tabular}


In the next sensitivity analysis, we removed the study by Zamboni and colleagues and changed zero to one in each cell for the study by Doepp and colleagues, thus providing the most conservative estimation of the odds of chronic cerebrospinal venous insufficiency in patients with multiple sclerosis versus healthy controls. This analysis yielded a substantially weaker but still statistically significant association compared with the primary analysis (OR 3.7, 95\% CI 1.2$11.0 ; I^{2}=72 \%$ ) (Figure $\mathrm{C}$ in Appendix 2).

The exclusion of studies that were reported as not blinded ${ }^{10,11,14}$ produced similar results to the primary analysis comparing patients with multiple sclerosis and healthy controls (OR 11.4, 95\% CI 1.8-73.2; $I^{2}=91 \%$ ) (Figure D in Appendix 2).

When we removed the study by Zamboni and colleagues from the meta-analysis comparing patients with multiple sclerosis and those with other neurologic diseases, the association was greatly weakened (OR 3.5, 95\% CI 0.8-15.8) and heterogeneity was reduced $\left(I^{2}=37 \%\right)$ (Figure $\mathrm{E}$ in Appendix 2).

When we assumed that all patients in the study by Zivadinov and colleagues ${ }^{16}$ (in whom ultrasound parameter two [reversal of flow in at least one of the deep cerebral veins] could not be assessed) were negative for that parameter, or when we removed patients with clinically isolated syndrome from the multiple sclerosis group, there was no material effect on the results.

\section{Interpretation}

We found a strong and statistically significant association between chronic cerebrospinal venous insufficiency and multiple sclerosis. However, the large amount of heterogeneity among the study results prevents a definitive conclusion. The source of the heterogeneity is not clear. It is not obviously caused by differences in the definition of chronic cerebrospinal venous insufficiency, patient characteristics or the methodologic quality of the studies.

We also could not identify any factor that accounted for the large and problematic difference between the studies in the frequency of chronic cerebrospinal venous insufficiency among patients with multiple sclerosis. Although small samples may be partly responsible, the magnitude of the difference strongly suggests that other factors are important. One obvious possibility is differences in ultrasound technique. It is crucial that ultrasonographers agree upon the technique to be used to diagnose chronic cerebrospinal venous insufficiency, and on a method to ensure quality control.

MS patients v. healthy controls

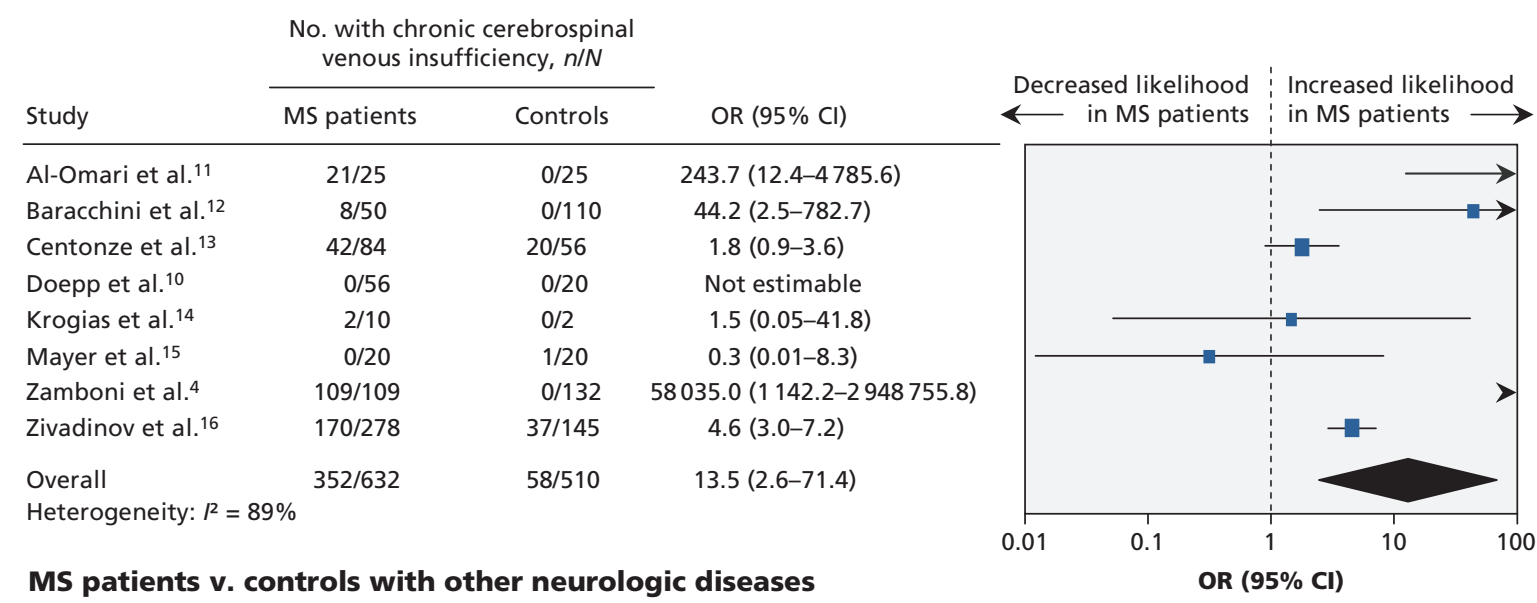

\begin{tabular}{lccc} 
Study & MS patients & Controls & OR $(95 \% \mathrm{Cl})$ \\
\hline Baracchini et al. ${ }^{12}$ & $8 / 50$ & $0 / 60$ & $24.2(1.4-430.7)$ \\
Krogias et al. $^{14}$ & $2 / 10$ & $0 / 5$ & $3.2(0.1-81.0)$ \\
Zamboni et al. $^{4}$ & $109 / 109$ & $0 / 45$ & $19929.0(389.5-1019755.6)$ \\
Zivadinov et al. $^{16}$ & $170 / 278$ & $11 / 24$ & $1.9(0.8-4.3)$ \\
Overall & $289 / 447$ & $11 / 134$ & $32.5(0.6-1775.7)$ \\
Heterogeneity: ${ }^{2}=90 \%$ & & &
\end{tabular}

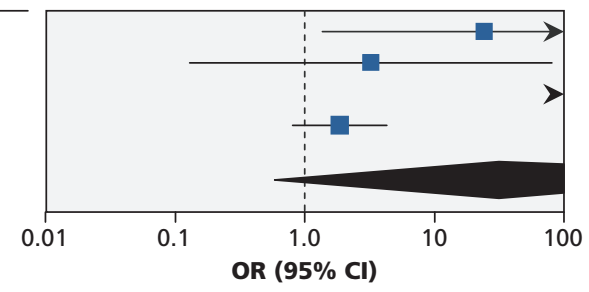

Figure 2: Meta-analysis of diagnosis of chronic cerebrospinal venous insufficiency (presence of at least two parameters) in patients with multiple sclerosis (MS) versus healthy controls (top panel) and controls with other neurologic diseases (bottom panel). An odds ratio greater than 1.0 indicates an increased likelihood of a diagnosis of chronic cerebrospinal venous insufficiency in MS patients versus controls. $\mathrm{Cl}=$ confidence interval, $\mathrm{OR}=$ odds ratio. 


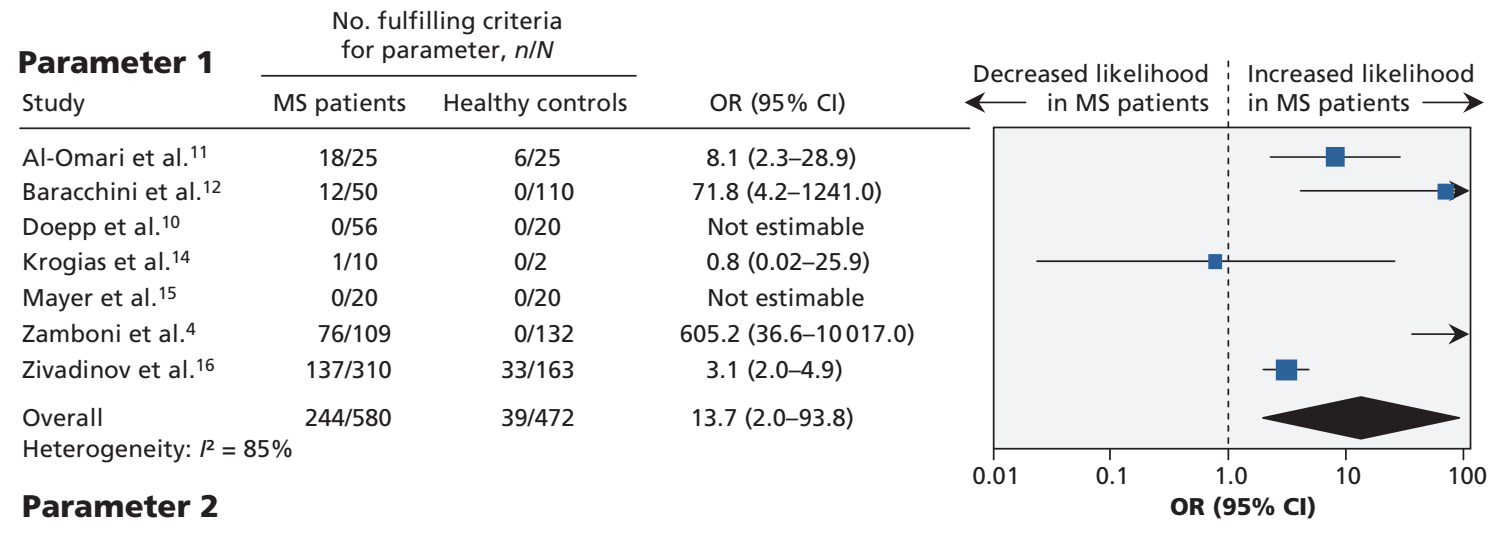

Study

\begin{tabular}{lccc}
\hline Baracchini et al. ${ }^{12}$ & $0 / 50$ & $0 / 110$ & Not estimable \\
Doepp et al. $^{10}$ & $1 / 56$ & $0 / 20$ & $1.1(0.04-28.3)$ \\
Krogias et al. $^{14}$ & $1 / 10$ & $0 / 2$ & $0.8(0.02-25.9)$ \\
Mayer et al. ${ }^{15}$ & $0 / 20$ & $0 / 20$ & Not estimable \\
Zamboni et al. ${ }^{4}$ & $55 / 109$ & $0 / 132$ & $269.9(16.4-4447.0)$ \\
Zivadinov et al. ${ }^{16}$ & $110 / 236$ & $15 / 118$ & $6.0(3.3-10.9)$ \\
Overall & $167 / 481$ & $15 / 402$ & $6.9(0.6-74.9)$ \\
Heterogeneity: $I^{2}=75 \%$ & & &
\end{tabular}

Heterogeneity: $I^{2}=75 \%$

Parameter 3

\begin{tabular}{lccc} 
Study & MS patients & Healthy controls & OR $(95 \% \mathrm{Cl})$ \\
\hline Baracchini et al. $^{12}$ & $8 / 50$ & $0 / 10$ & $44.2(2.5-782.7)$ \\
Doepp et al. ${ }^{10}$ & $0 / 56$ & $0 / 20$ & Not estimable \\
Krogias et al. ${ }^{14}$ & $0 / 10$ & $0 / 2$ & Not estimable \\
Mayer et al. ${ }^{15}$ & $13 / 20$ & $16 / 20$ & $0.5(0.1-1.9)$ \\
Zamboni et al. ${ }^{4}$ & $30 / 109$ & $1 / 177$ & $66.8(9.0-498.8)$ \\
Zivadinov et al. ${ }^{16}$ & $197 / 310$ & $63 / 163$ & $2.8(1.9-4.1)$ \\
Overall & $248 / 555$ & $80 / 492$ & $6.0(0.9-40.3)$ \\
Heterogeneity: $I^{2}=86 \%$ & & &
\end{tabular}

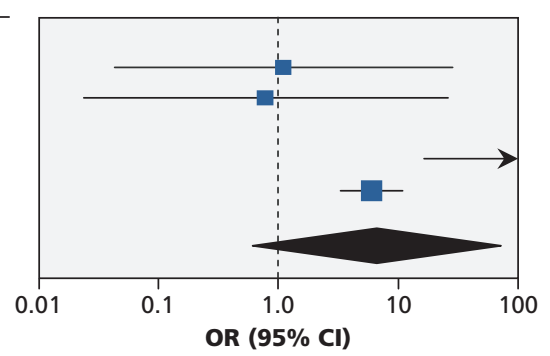

\section{Parameter 4}

Study

\begin{tabular}{|c|c|c|c|}
\hline Al-Omari et al. ${ }^{11}$ & $0 / 25$ & $0 / 25$ & Not estimable \\
\hline Baracchini et al. ${ }^{12}$ & $3 / 50$ & $1 / 110$ & $7.0(0.7-68.6)$ \\
\hline Doepp et al. ${ }^{10}$ & $5 / 56$ & $1 / 20$ & $1.9(0.2-17.0)$ \\
\hline Krogias et al. ${ }^{14}$ & $3 / 10$ & $0 / 2$ & $2.3(0.1-62.7)$ \\
\hline Mayer et al. ${ }^{15}$ & $0 / 20$ & $1 / 20$ & $0.3(0.01-8.3)$ \\
\hline Zamboni et al. 4 & $35 / 109$ & $1 / 177$ & $83.2(11.2-618.9$ \\
\hline Zivadinov et al. ${ }^{16}$ & $30 / 310$ & $12 / 163$ & $1.4(0.7-2.7)$ \\
\hline Overall & $76 / 580$ & $16 / 517$ & $3.5(0.7-18.5)$ \\
\hline
\end{tabular}

Heterogeneity: $I^{2}=76 \%$

\section{Parameter 5}

Study

\begin{tabular}{lccc} 
Study & MS patients & Healthy controls & OR $(95 \% \mathrm{Cl})$ \\
\hline Al-Omari et al. ${ }^{11}$ & $4 / 25$ & $0 / 25$ & $10.7(0.5-209.6)$ \\
Baracchini et al. ${ }^{12}$ & $8 / 50$ & $6 / 110$ & $3.3(1.1-10.1)$ \\
Doepp et al. ${ }^{10}$ & $4 / 56$ & $3 / 20$ & $0.4(0.1-2.2)$ \\
Mayer et al. ${ }^{15}$ & $0 / 20$ & $0 / 20$ & Not estimable \\
Zamboni et al. $^{4}$ & $61 / 109$ & $21 / 177$ & $9.4(5.2-17.1)$ \\
Zivadinov et al. ${ }^{16}$ & $35 / 310$ & $11 / 163$ & $1.8(0.9-3.6)$ \\
Overall & $112 / 570$ & $41 / 515$ & $2.9(0.96-8.6)$ \\
Heterogeneity: $I^{2}=81 \%$ & & &
\end{tabular}

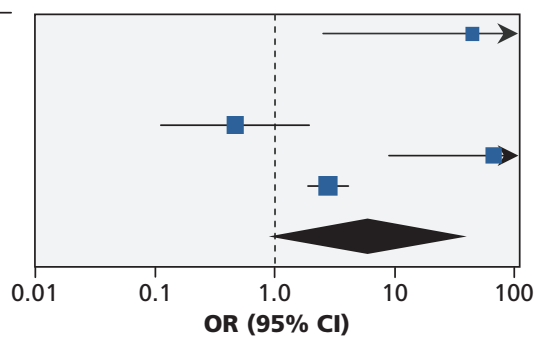

Heterogeneity: $l^{2}=81 \%$
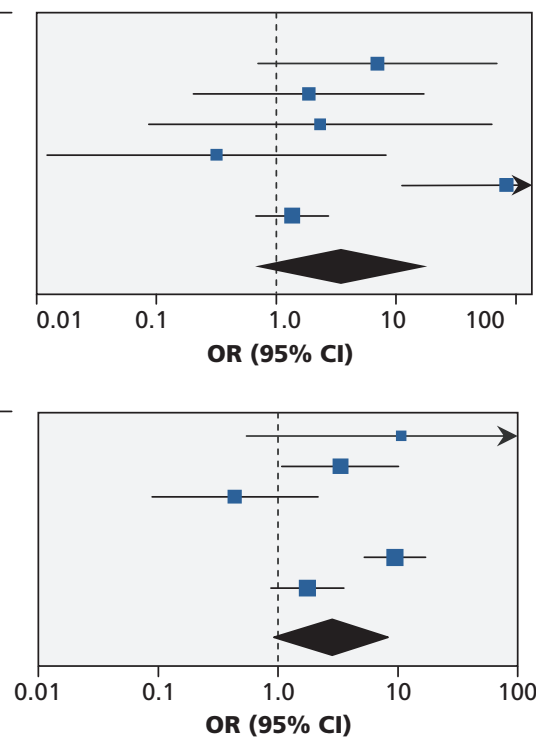

Figure 3: Meta-analysis of individual parameters of chronic cerebrospinal venous insufficiency in patients with multiple sclerosis (MS) versus healthy controls. (1) Reflux in internal jugular veins or vertebral veins; (2) flow reversal in deep cerebral veins; (3) stenoses of internal jugular vein; (4) flow not detectable in internal jugular veins or vertebral veins; and (5) reverted postural control of main cerebral venous outflow pathway (internal jugular veins). An odds ratio greater than 1.0 indicates an increased likelihood of the parameter being present in $\mathrm{MS}$ patients versus controls. $\mathrm{Cl}=$ confidence interval, $\mathrm{OR}=$ odds ratio. 
Reflux in the deep cerebral veins is the most technically challenging of the ultrasound parameters to identify. One study was unable to assess it at all, ${ }^{11}$ and another was unable to assess it in $28 \%$ of patients. ${ }^{16}$ Another study, which we excluded from our systematic review because it did not have a control group of people without multiple sclerosis, was also unable to evaluate the deep cerebral veins. ${ }^{17}$ This questions the appropriateness of having a diagnostic parameter that is difficult to assess and emphasizes the importance of training ultrasonographers in how to assess the deep cerebral veins.

Because ultrasonography is so operatordependent, the lack of blinding in three studies, and the lack of a description of how well blinding was achieved in all of the other studies, is of concern. Future studies should report the mechanism and success of blinding in detail. The intra- and interobserver variability of the diagnosis of chronic cerebrospinal venous insufficiency also needs assessment. Zivadinov and

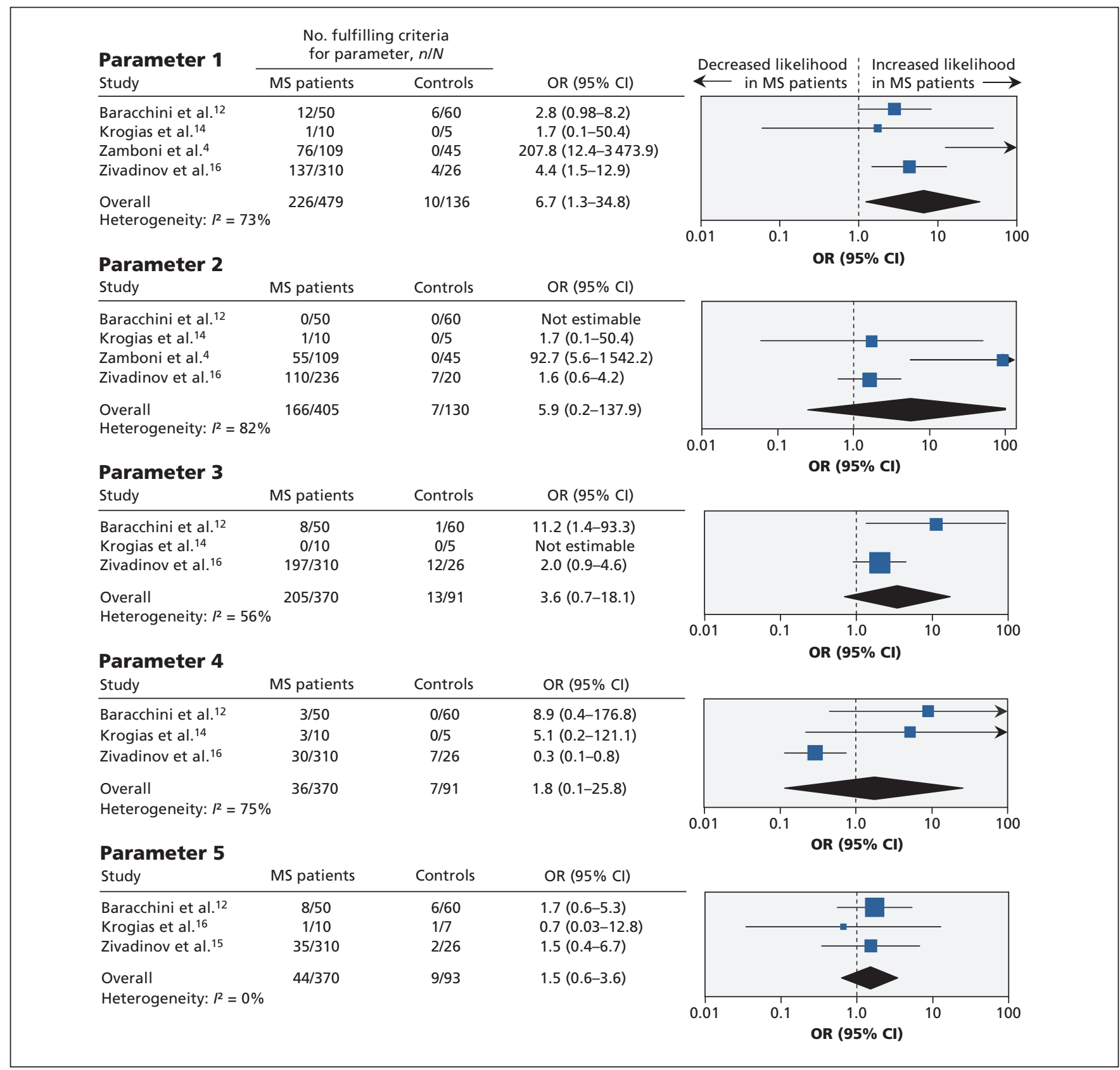

Figure 4: Meta-analysis of individual parameters of chronic cerebrospinal venous insufficiency in patients with multiple sclerosis (MS) versus controls with other neurologic diseases. (1) Reflux in internal jugular veins or vertebral veins; (2) flow reversal in deep cerebral veins; (3) stenoses of internal jugular vein; (4) flow not detectable in internal jugular veins or vertebral veins; and (5) reverted postural control of main cerebral venous outflow pathway (internal jugular veins). An odds ratio greater than 1.0 indicates an increased likelihood of the parameter being present in MS patients versus controls. $\mathrm{Cl}=$ confidence interval, $\mathrm{OR}=$ odds ratio. 
colleagues assessed the intrarater reliability of the diagnosis of the condition in 28 patients (11 with multiple sclerosis) one week apart and found an agreement of $89 \%$ and a kappa value of $0.75 .{ }^{16}$ The diagnosis differed between raters in $11 \%(3 / 28)$ of patients. Another paper about reproducibility did not provide data in a manner allowing interpretation similar to the above. ${ }^{18}$ More studies of the reproducibility of the assessment of chronic cerebrospinal venous insufficiency are needed.

An association between chronic cerebrospinal venous insufficiency and multiple sclerosis does not mean that the condition causes multiple sclerosis. Indeed, some observations, including a subgroup analysis of the methodologically sound study by Zivadinov and colleagues, ${ }^{16}$ suggest that chronic cerebrospinal venous insufficiency may be a consequence of multiple sclerosis. In the study by Zivadinov and colleagues, the frequency of chronic cerebrospinal venous insufficiency was roughly inversely correlated to the duration of multiple sclerosis: $38 \%$ (8/21) among patients with clinically isolated syndrome, 49\% (94/191) among those with relapsing-remitting multiple sclerosis and 73\% (58/80) among those with secondary progressive multiple sclerosis. Another study, which used angiography in 42 patients with multiple sclerosis, found stenosis of the jugular or azygos vein in 9\% (1/11) of patients with clinically isolated syndrome, 33\% (6/18) of those with early relapsing-remitting multiple sclerosis and $89 \%$ (12/13) of those with late multiple sclerosis (average duration 14 years). ${ }^{19}$ These observations are not definitive, and further research examining the link between chronic cerebrospinal venous insufficiency and duration of multiple sclerosis is needed.

\section{Limitations}

Only eight studies were eligible for our review, and many of them were small. Three studies were not blinded, and none of the others reported the success of blinding. The description of the ultrasound technique was limited in some studies, so it is unclear whether the techniques differed across the studies. As with most systematic reviews, there is the potential for publication bias. However, the question about whether chronic cerebrospinal venous insufficiency is associated with multiple sclerosis is so controversial that we feel positive and negative studies are both likely to be published. Finally, we did not have access to individual patient data and therefore were unable to explore whether the frequency of chronic cerebrospinal venous insufficiency was affected by individual factors such as age, sex and type of multiple sclerosis.

\section{Conclusion}

We found a strong association between chronic cerebrospinal venous insufficiency and multiple sclerosis. However, poor reporting of the success of blinding and marked heterogeneity among the studies included in our review precluded definitive conclusions. Further high-quality studies, using identical ultrasound protocols, are needed to definitively determine whether chronic cerebrospinal venous insufficiency is more frequent among patients with multiple sclerosis than among those without it. Our meta-analysis will need to be updated as the results of future studies become available.

\section{References}

1. Bar-Or A. The immunology of multiple sclerosis. Semin Neurol 2008;28:29-45.

2. Zamboni P. The big idea - iron-dependent inflammation in venous disease and proposed parallels in multiple sclerosis. $J R$ Soc Med 2006;99:589-93.

3. Zamboni P, Galeotti R, Menegatti E, et al. Chronic cerebrospinal venous insufficiency in patients with multiple sclerosis. J Neurol Neurosurg Psychiatry 2009;80:392-9.

4. Zamboni P, Menegatti E, Galeotti R, et al. The value of cerebral Doppler venous haemodynamics in the assessment of multiple sclerosis. J Neurol Sci 2009;282:21-7.

5. Bartolomei I, Salvi F, Galeotti R, et al. Hemodynamic patterns of chronic cerebrospinal venous insufficiency in multiple sclerosis. Correlation with symptoms at onset and clinical course. Int Angiol 2010;29:183-8.

6. Khan O, Filippi M, Freedman MS, et al. Chronic cerebrospinal venous insufficiency and multiple sclerosis. Ann Neurol 2010;67: 286-90.

7. Worthington V, Kilestein J, Eikelenboom MJ, et al. Normal CSF ferritin levels in MS suggest against etiologic role of chronic venous insufficiency. Neurology 2010;75:1617-22.

8. Wells GA, Shea B, O'Connell D, et al. The Newcastle-Ottawa Scale (NOS) for assessing the quality of non-randomised studies in meta-analyses. Ottawa (ON): The Ottawa Hospital Research Institute. Available: www.ohri.ca/programs/clinical_epidemiology /oxford.asp (accessed 2011 June 25).

9. Higgins JPT, Green S, editors. Cochrane handbook for systematic reviews of interventions. Version 5.1.0. The Cochrane Collaboration; 2011. Available: www.cochrane-handbook.org (accessed 2011 June 25).

10. Doepp F, Paul F, Valdueza JM, et al. No cerebrocervical venous congestion in patients with multiple sclerosis. Ann Neurol 2010; 68:173-83.

11. Al-Omari MH, Rousan LA. Internal jugular vein morphology and hemodynamics in patients with multiple sclerosis. Int Angiol 2010;29:115-20.

12. Baracchini C, Perini P, Calabrese M, et al. No evidence of chronic cerebrospinal venous insufficiency at multiple sclerosis onset. Ann Neurol 2011;69:90-9.

13. Centonze D, Floris R, Stefanini M, et al. Proposed chronic cerebrospinal venus insufficiency criteria do not predict MS risk nor MS severity. Ann Neurol 2011;70:51-8.

14. Krogias C. Schroder A, Wiendl H, et al. "Chronic cerebrospinal venous insufficiency" and multiple sclerosis: critical analysis and first observation in an unselected cohort of MS patients [article in German]. Nervenarzt 2010;81:740-6.

15. Mayer CA, Pfeilschifter W, Lorenz MW, et al. The perfect crime? Chronic cerebrospinal venous insufficiency not leaving a trace in MS. J Neurol Neurosurg Psychiatry 2011;82:436-40.

16. Zivadinov R, Marr K, Cutter G, et al. Prevalence, sensitivity, and specificity of chronic cerebrospinal venous insufficiency in MS. Neurology 2011;77:138-4.

17. Simka M, Kostecki J, Zaniewski M, et al. Extracranial Doppler sonographic criteria of chronic cerebrospinal venous insufficiency in the patients with multiple sclerosis. Int Angiol 2010;29:109-14.

18. Menegatti E, Genova V, Tessari M, et al. The reproducibility of colour Doppler in chronic cerebrospinal venous insufficiency associated with multiple sclerosis. Int Angiol 2010;29:121-6.

19. Yamout B, Herlopian A, Issa Z, et al. Extracranial venous stenosis is an unlikely cause of multiple sclerosis. Mult Scler 2010; 16:1341-8. 
Affiliations: From the Keenan Research Centre (Laupacis, Lillie, Straus, Perrier, Thorpe, Spears), Li Ka Shing Knowledge Institute of St. Michael's Hospital, Toronto, Ont.; the Faculty of Medicine (Laupacis, Dueck, Straus, Aviv, Spears), University of Toronto, Toronto, Ont.; the Schulich Heart Centre (Dueck), Sunnybrook Health Sciences Centre, Toronto, Ont.; the Office of Continuing Education and Professional Development (Perrier), Faculty of Medicine, University of Toronto, Toronto, Ont.; the Department of Clinical Neurosciences (Burton), Hotchkiss Brain Institute, University of Calgary, Calgary, Alta.; the Department of Medical Imaging (Aviv), Sunnybrook Health Sciences Centre, Toronto, Ont.; the Dalla Lana School of Public Health (Thorpe), University of Toronto, Toronto, Ont.; and the Faculty of Medicine (Feasby), University of Calgary, Calgary. Alta.

Contributors: Andreas Laupacis, Andrew Dueck, Sharon Straus, Laure Perrier, Jodie Burton, Richard Aviv, Kevin Thorpe, Thomas Feasby and Julian Spears designed the study.
Erin Lillie drafted the systematic review protocol, and Laure Perrier conducted the literature searches. Andreas Laupacis and Sharon Straus participated in screening articles for review. Andreas Laupacis, Erin Lillie, Andrew Dueck and Laure Perrier contributed to data abstraction. Andreas Laupacis and Kevin Thorpe supervised the analysis. Erin Lillie performed the meta-analyses and sensitivity analyses, and Andrew Dueck provided input to the sensitivity analyses. Andreas Laupacis drafted the manuscript. All of the authors revised the manuscript and approved the final version submitted for publication.

Funding: Funding for this study was provided by the Canadian Institutes of Health Research (grant no. 243347). The study sponsors had no role in the design of the study, the collection, analysis or interpretation of the data, the writing of the report, or the decision to submit the manuscript for publication.

Acknowledgement: We thank Andrea Tricco for her help with the systematic review. 